\title{
ABOUT THE STUDY OF REQUESTS BUCKLING VERIFICATION OF THE ELEMENTS OF POWER PLANT PROPULSION
}

\author{
Anastase PRUIU ${ }^{1}$ \\ Daniel MARASESCU ${ }^{2}$ \\ Dorin-Silviu BANU ${ }^{3}$ \\ Ion Adrian GIRBA ${ }^{4}$ \\ ${ }^{1}$ Professor PhD Eng., Marine Engineering and Naval Weapons Department \\ ${ }^{2} \mathrm{PhD}$ attendee Eng., Marine Engineering and Naval Weapons Department \\ ${ }^{3} \mathrm{PhD}$ attendee Eng. Military Technical Academy \\ ${ }^{2} \mathrm{PhD}$ attendee Eng. Military Technical Academy
}

\begin{abstract}
On study based of requests from the power propulsion plant, the authors develop in this paper a study of buckling heat engine piston rod at the head of the cross, the strut rod and shaft intermediaries for power propulsion plant.
\end{abstract}

Key words: buckling, strut rod, propulsion power plant

\section{Introduction}

For parts-metallic structures of naval propulsion plants, compression can be applied to situations of exploitation when axial forces grow, the issue of verification of buckling.

This consists of:

a. determining the critical buckling force

b. determining the critical buckling pressure

c. determination the coefficient of safety buckling

d. depending on the results obtained can take precautions to avoid operational achieving critical force buckling

e. resizing piece to increase the safety coefficient buckling

Buckling phenomenon is dangerous because it is irreversible.

Critical buckling tension may fall to limit proportionalities (elasticity) of the material from which it is made corresponding to the piece, is called elastic buckling.

If the tension is above the critical elastic buckling, buckling is called the elastic-plastic.

Either: $\quad \mathrm{F}_{\mathrm{crf}}[\mathrm{kN}]$ - critical buckling force

$\mathrm{F}_{\text {ef }}[\mathrm{kN}]$ - effective buckling force

The safety coefficient of buckling is determined with:

with the condition like $c_{f}>1$

$$
c_{f}=\frac{\mathrm{F}_{\text {crf }}}{\mathrm{F}_{\mathrm{eff}}}[1]
$$

Depending on the functional importance of the piece of naval propulsion power plant, buckling safety coefficient will be accepted in a particular reference domain.

Critical buckling force

$$
F_{c r f}=\frac{\pi^{2} \cdot E \cdot I_{\min }}{u_{f}^{2}}[\mathrm{KN}]
$$

$E\left[\mathrm{kN} / \mathrm{m}^{2}\right]$ - elastic modulus specific for the material it is made subjected to compressive load the piece. For the manufacture of the materials used in naval propulsion power plant, determination of the elastic modulus should be performed in the material strength laboratories recognized by classification societies and for materials recognize by them.

$I_{\min }\left[\mathrm{m}^{4}\right]$ - moment of inertia calculated for minimal section of the piece put under compression. This is calculated based on the geometric size and shape of the respective section.

$\mathrm{L}_{\mathrm{f}}^{2}\left[\mathrm{~m}^{2}\right]$ - buckling length is determined depending on the length and attachment, assembling piece in the system.

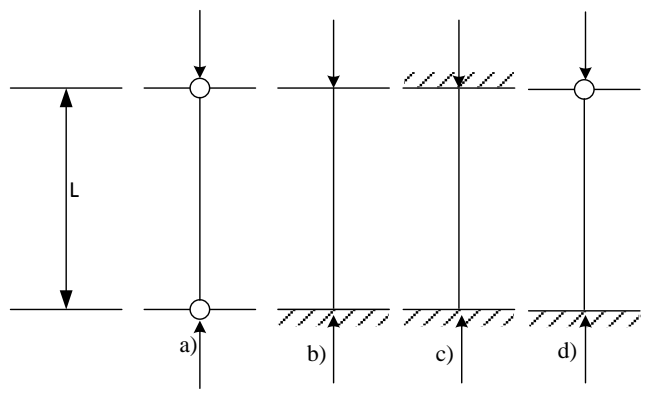

Fig. 1. Modes of assembling the piece

$\mathrm{L}$ - length of piece, a) double articulated bar, b) embedded bar,

c) double embedded bar, d) embedded and articulated bar

For this cases results:
a) $L_{f}=L$
b) $L_{f}=2 x L$
c) $L_{f}=L / 2$
d) $L_{f}=0.707 \times L$ 
"Mircea cel Batran" Naval Academy Scientific Bulletin, Volume XIX - 2016 - Issue 2

The journal is indexed in: PROQUEST / DOAJ / Crossref / EBSCOhost / INDEX COPERNICUS / DRJI / OAJI I JOURNAL INDEX I I2OR / SCIENCE LIBRARY INDEX / Google Scholar / Academic Keys/ ROAD Open Access I Academic Resources / Scientific Indexing Services / SCIPIO / JIFACTOR

For fixing arrangement are taken into account two perpendicular planes of section fixing joints, otherwise for articulated plan $L_{f}=L$, but for the normally plan be considered embedded, so $L_{f}=$ $\mathrm{L} / 2$ what will lead to the determination of two critical forces buckling.

$F_{\text {crf (PO) }}[\mathrm{kN}]$ - critical buckling force for articulated plan - called oscillation plan

$\mathrm{F}_{\mathrm{crf}(\mathrm{PI})}[\mathrm{kN}]$ - critical buckling force for normal plan on oscillation plan - called embedded plan

To equalize the two critical buckling forces, proceed to mass redistribution of minimum calculation section so:

$$
I_{\min (\mathrm{PO})}=4 \times I_{\min (\mathrm{PI})}
$$

By equalizing critical safety buckling forces is obtained equalization of critical safety buckling coefficients for both plans assembling piece

\section{B.1.Checking the buckling of piston rod}

\begin{tabular}{|c|c|c|}
\hline $\mathrm{D}[\mathrm{m}]$ & 0.5 & 0.72 \\
\hline $\mathrm{S}_{\mathrm{p}}\left[\mathrm{m}^{2}\right]$ & 0.196 & 0.407 \\
\hline $\mathrm{d}_{\mathrm{TP}}[\mathrm{m}]$ & 0.15 & 0.216 \\
\hline $\mathrm{S}[\mathrm{m}]$ & 2 & 3 \\
\hline $\mathrm{L}_{\mathrm{TP}}[\mathrm{m}]$ & 3 & 4.5 \\
\hline$E\left[\mathrm{kN} / \mathrm{m}^{2}\right]$ & $2.1 \times 10^{8}$ & $2.1 \times 10^{8}$ \\
\hline $\mathrm{I}_{\min }\left[\mathrm{m}^{4}\right]$ & $2.48 \times 10^{-5}$ & $1.068 \times 10^{-4}$ \\
\hline $\mathrm{p}_{\text {maxaid }}[\mathrm{bar}]$ & 135 & 140 \\
\hline $\mathrm{p}_{\text {maxaid }}\left[\mathrm{kN} / \mathrm{m}^{2}\right]$ & $135 \times 10^{2}$ & $140 \times 10^{2}$ \\
\hline $\mathrm{p}_{\text {maxdss }}[$ bar] & 150 & 155 \\
\hline $\begin{array}{l}\mathrm{p}_{\text {maxdss }} \\
{\left[\mathrm{kN} / \mathrm{m}^{2}\right]}\end{array}$ & $150 \times 10^{2}$ & $155 \times 10^{2}$ \\
\hline $\mathrm{F}_{\mathrm{pmax}}[\mathrm{kN}]$ & $29.4 \times 10^{2}$ & $63 \times 10^{2}$ \\
\hline $\mathrm{F}_{\text {crf }}[\mathrm{kN}]$ & $2.28 \times 10^{4}$ & $4.373 \times 10^{4}$ \\
\hline $\mathrm{C}_{\mathrm{f}}[-]$ & 7.75 & 6.941 \\
\hline
\end{tabular}

B.2. Checking the buckling of piston rod

\begin{tabular}{|l|l|l|}
\hline $\mathrm{D}[\mathrm{m}]$ & 0.84 & 1.08 \\
\hline $\mathrm{S}_{\mathrm{p}}\left[\mathrm{m}^{2}\right]$ & 0.554 & 0.855 \\
\hline $\mathrm{d}_{\mathrm{TP}}[\mathrm{m}]$ & 0.25 & 0.32 \\
\hline $\mathrm{S}[\mathrm{m}]$ & 3.2 & 2.8 \\
\hline $\mathrm{L}_{\mathrm{TP}}[\mathrm{m}]$ & 5 & 5 \\
\hline $\mathrm{E}\left[\mathrm{kN} / \mathrm{m}^{2}\right]$ & $2.1 \times 10^{8}$ & $2.1 \times 10^{8}$ \\
\hline $\mathrm{I}_{\min }\left[\mathrm{m}^{4}\right]$ & $1.91 \times 10^{-4}$ & $5.044 \times 10^{-4}$ \\
\hline $\mathrm{p}_{\operatorname{maxaid}}[\mathrm{bar}]$ & 140 & 140 \\
\hline $\mathrm{p}_{\operatorname{maxaid}}\left[\mathrm{kN} / \mathrm{m}^{2}\right]$ & $140 \times 10^{2}$ & $140 \times 10^{2}$ \\
\hline $\mathrm{p}_{\operatorname{maxdss}}[\mathrm{bar}]$ & 155 & 155 \\
\hline $\begin{array}{l}\mathrm{p}_{\operatorname{maxdss}} \\
{\left[\mathrm{kN} / \mathrm{m}^{2}\right]}\end{array}$ & $155 \times 10^{2}$ & $155 \times 10^{2}$ \\
\hline $\mathrm{F}_{\mathrm{pmax}}[\mathrm{kN}]$ & $85.87 \times 10^{2}$ & $132 \times 10^{2}$ \\
\hline $\mathrm{F}_{\mathrm{crf}}[\mathrm{kN}]$ & $6.33 \times 10^{4}$ & $16.73 \times 10^{4}$ \\
\hline $\mathrm{c}_{\mathrm{f}}[-]$ & 7.38 & 12.6 \\
\hline
\end{tabular}

$\mathrm{D}[\mathrm{m}]$ - cylinder diameter; $\mathrm{S}_{\mathrm{p}}\left[\mathrm{m}^{2}\right]$ - piston area; $\mathrm{d}_{\mathrm{TP}}[\mathrm{m}]$ - rod of piston diameter; $\mathrm{S}$ [m] - piston stroke; $L_{T P}[\mathrm{~m}]$ - rod of piston length; $I_{\min }\left[\mathrm{m}^{4}\right]$ inertia moment; $p_{\text {maxard }}\left[\mathrm{kN} / \mathrm{m}^{2}\right]$-maximum combustion pressure; $p_{\text {maxdss }}\left[\mathrm{kN} / \mathrm{m}^{2}\right]-$ maximum pressure for safety valve; $F_{p m a x}[k N]$ - maximum forte of pressure; $\sigma_{\text {efmax }}\left[\mathrm{kN} / \mathrm{m}^{2}\right]$ - maximum effective tension; $F_{\text {crf }}[\mathrm{kN}]$ - critical force buckling; $\sigma_{\text {crf }}\left[\mathrm{kN} / \mathrm{m}^{2}\right]$ - critical tension buckling; $\mathrm{c}_{\mathrm{f}}[-]$ buckling safety factor;

$$
\begin{aligned}
& S_{p}=\frac{\pi \cdot D^{2}}{4}\left[m^{2}\right] ; \quad \text { [4] } \\
& I_{\min }=\frac{\pi \cdot d_{T P}^{2}}{64}\left[m^{4}\right] ; \quad \text { [5] } \\
& F_{\text {pmax }}=p_{\operatorname{maxdsg}} \times \frac{\pi D^{2}}{4}[\mathrm{kN}] ;[6] \\
& \sigma_{\text {efmax }}=\frac{E_{\text {pmax }}}{\frac{\pi \cdot d_{f P}^{2}}{4}}\left[\frac{k W}{m^{2}}\right] \text {; } \\
& F_{c r f}=\frac{\pi^{2} \cdot E \cdot I_{\min }}{\left(\frac{L_{T r}}{2}\right)^{2}}[\mathrm{kN}] \\
& \sigma_{c r f}=\frac{\sigma_{c r f}^{2}}{\frac{\pi \cdot d_{T P}^{2}}{s}}\left[\frac{\mathrm{kN}}{\mathrm{m}^{2}}\right] \text {; } \\
& c_{f}=\frac{F_{c r f}}{F_{p \max }}=\frac{\sigma_{c r f}}{\sigma_{\text {sf } \max }} ;
\end{aligned}
$$

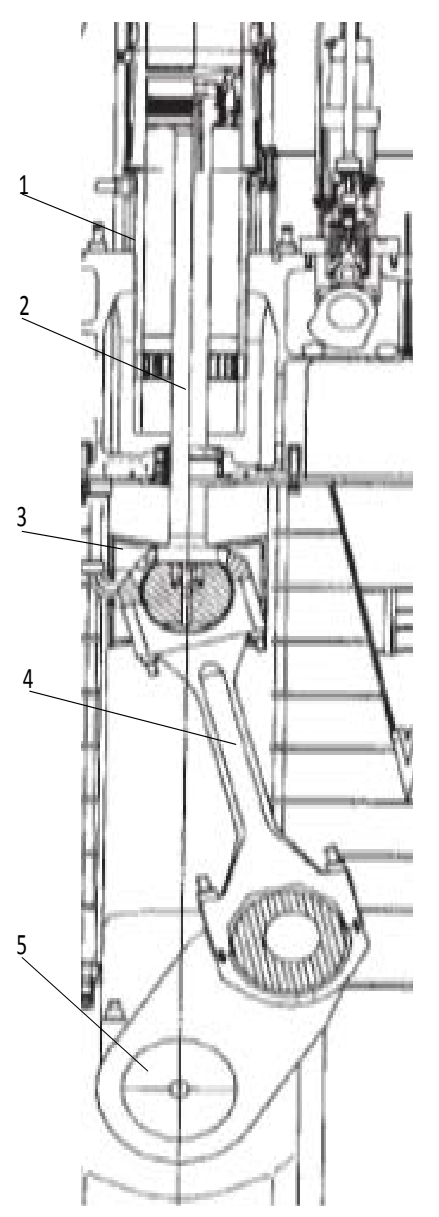

Fig.2 piston rod and connecting rod for MAN S46 [B6]

1 - piston, 2 - piston rod,

3 - cross head, 4 - connecting rod,

5 - crankshaft 
"Mircea cel Batran" Naval Academy Scientific Bulletin, Volume XIX - 2016 - Issue 2 The journal is indexed in: PROQUEST / DOAJ / Crossref / EBSCOhost / INDEX COPERNICUS / DRJI / OAJI I JOURNAL INDEX I I2OR / SCIENCE LIBRARY INDEX / Google Scholar / Academic Keys/ ROAD Open Access I Academic Resources / Scientific Indexing Services / SCIPIO / JIFACTOR

\section{C.1. Checking the buckling of connectingstrut} rod

\begin{tabular}{|l|l|l|}
\hline $\mathrm{D}[\mathrm{m}]$ & 0.5 & 0.72 \\
\hline $\mathrm{S}[\mathrm{m}]$ & 2 & 3 \\
\hline $\mathrm{L}_{\mathrm{TB}}[\mathrm{m}]$ & 2.63 & 3.95 \\
\hline $\mathrm{F}_{\text {pmaxdss }}[\mathrm{kN}]$ & $29.4 \times 10^{2}$ & $63 \times 10^{2}$ \\
\hline $\mathrm{E}\left[\mathrm{kN} / \mathrm{m}^{2}\right]$ & $2.1 \times 10^{8}$ & $2.1 \times 10^{8}$ \\
\hline $\mathrm{d}_{\mathrm{TB}}[\mathrm{m}]$ & 0.18 & 0.22 \\
\hline $\mathrm{I}_{\min }\left[\mathrm{m}^{4}\right]$ & $5.15 \times 10^{-5}$ & $1.27 \times 10^{-4}$ \\
\hline $\mathrm{F}_{\text {crf PO }}[\mathrm{kN}]$ & $15.43 \times 10^{3}$ & $1.688 \times 10^{4}$ \\
\hline $\mathrm{C}_{\mathrm{fPO}}[-]$ & 5.25 & 2.679 \\
\hline $\mathrm{F}_{\text {crf PI }}[\mathrm{kN}]$ & $6172 \times 10^{3}$ & $6.72 \times 10^{4}$ \\
\hline $\mathrm{C}_{\mathrm{fPI}}[-]$ & 21 & 10.716 \\
\hline
\end{tabular}

C.2. Checking the buckling of connecting strut rod

\begin{tabular}{|l|l|l|}
\hline $\mathrm{D}[\mathrm{m}]$ & 0.84 & 1.08 \\
\hline $\mathrm{S}[\mathrm{m}]$ & 3.2 & 2.8 \\
\hline $\mathrm{L}_{\mathrm{TB}}[\mathrm{m}]$ & 4.21 & 4 \\
\hline $\mathrm{F}_{\mathrm{pmaxdss}}[\mathrm{kN}]$ & $85.87 \times 10^{2}$ & $132 \times 10^{2}$ \\
\hline $\mathrm{E}\left[\mathrm{kN} / \mathrm{m}^{2}\right]$ & $2.1 \times 10^{8}$ & $2.1 \times 10^{8}$ \\
\hline $\mathrm{d}_{\mathrm{TB}}[\mathrm{m}]$ & 0.28 & 0.35 \\
\hline $\mathrm{I}_{\min }\left[\mathrm{m}^{4}\right]$ & $3.01 \times 10^{-4}$ & $7.37 \times 10^{-4}$ \\
\hline $\mathrm{F}_{\text {crf PO }}[\mathrm{kN}]$ & $3.52 \times 10^{4}$ & $9.55 \times 10^{4}$ \\
\hline $\mathrm{C}_{\mathrm{fPO}}[-]$ & 4.099 & 7.23 \\
\hline $\mathrm{F}_{\text {crf PI }}[\mathrm{kN}]$ & $14.08 \times 10^{4}$ & $38.2 \times 10^{4}$ \\
\hline $\mathrm{C}_{\mathrm{fPI}}[-]$ & 16.39 & 28.92 \\
\hline
\end{tabular}

$\mathrm{L}_{\text {тв }}[\mathrm{m}]$ - connecting rod length; $\lambda=0.25 \div 0.38$; $\lambda=\frac{S}{2 \cdot L_{T B}} ; \mathrm{d}_{\mathrm{TB}}-$ connecting rod diameter;

$\mathrm{F}_{\mathrm{crf}}(\mathrm{PO})[\mathrm{kN}]$ - critical buckling force for connecting rod oscillation plan;

$F_{\text {crf (PI) }}[\mathrm{kN}]$ - critical buckling force for connecting rod embedded plan;

$\sigma_{\mathrm{crt}(\mathrm{PO})}\left[\mathrm{kN} / \mathrm{m}^{2}\right]$ - critical tension buckling for connecting rod oscillation plan;

$\sigma_{\text {crr(PI) }}\left[\mathrm{kN} / \mathrm{m}^{2}\right]$ - critical tension buckling for connecting rod embedded plan;

$\mathrm{C}_{\mathrm{f}(\mathrm{PO})}[-]$ - buckling safety factor for connecting rod oscillation plan;

$\mathrm{C}_{\mathrm{f}(\mathrm{PI})}[-]$ - buckling safety factor for connecting rod embedded plan;

$$
\begin{aligned}
& F_{c r f(P O)}=\frac{\pi^{2} \cdot \mathbb{E} \cdot I_{\min }}{\mathbb{L}_{T B}^{2}}[\mathrm{kN}] ; \\
& F_{c r f(P D)}=\frac{\pi^{2} \cdot E_{I} \cdot U_{\min }}{\left(\frac{L_{T g}}{2}\right)^{2}}[\mathrm{kN}] ; \\
& I_{\min }=\frac{\pi \cdot d_{T B}^{2}}{64}\left[\mathrm{~m}^{4}\right] ;
\end{aligned}
$$

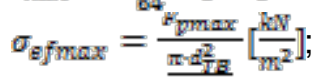

$$
\begin{aligned}
& \sigma_{c r f(P O)}=\frac{F_{c r f(P D)}^{4}}{\frac{\pi \cdot d_{T S}^{2}}{4}}\left[\frac{\mathrm{kN}}{\mathrm{m}^{2}}\right] ;
\end{aligned}
$$

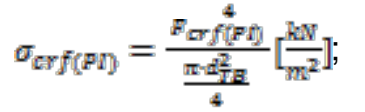

$$
\begin{aligned}
& c_{f(P O)}=\frac{F_{c r f(P D))}}{F_{\max }}=\frac{\sigma_{c r f(P O)}}{\sigma_{\mathrm{g} f \max }} ; \\
& c_{f(P D)}=\frac{F_{c r f(P t)}}{F_{p \max }}=\frac{\sigma_{c r f(P t)}}{\sigma_{\mathrm{affmax}}} ;
\end{aligned}
$$

\section{Material limitations [B4]}

Where shafts may experience vibratory stresses close to the permissible stresses for transient operation, the materials are to have a specified minimum ultimate tensile strength $\left(\sigma_{B}\right)$ of 500 $\mathrm{N} / \mathrm{mm}^{2}$. Otherwise materials having a specified minimum ultimate tensile strength () of $400 \mathrm{~N} / \mathrm{mm}^{2}$ may be used.

For use in the following formulae in this UR, $\sigma_{B}$ is limited as follows:

- For carbon and carbon manganese steels, a minimum specified tensile strength not exceeding $600 \mathrm{~N} / \mathrm{mm}^{2}$ for use in M68.5 and not exceeding $760 \mathrm{~N} / \mathrm{mm}^{2}$ in M68.4.

- For alloy steels, a minimum specified tensile strength not exceeding $800 \mathrm{~N} / \mathrm{mm}^{2}$.

- For propeller shafts in general a minimum specified tensile strength not exceeding 600 $\mathrm{N} / \mathrm{mm}^{2}$ (for carbon, carbon manganese and alloy steels).

Where materials with greater specified or actual tensile strengths than the limitations given above are used, reduced shaft dimensions or higher permissible vibration stresses are not acceptable when derived from the formulae in this UR.

\section{Shaft diameters [B4]}

Shaft diameters are not to be less than that determined from the following formula:

$$
d=F \times k \times \sqrt[3]{\frac{p}{n_{0}} \times \frac{1}{1-\frac{d_{k}^{4}}{d f_{0}^{4}} \times \frac{560}{\sigma_{B}+160}}}[\mathrm{~mm}]
$$

where:

$\mathrm{d}=$ minimum required diameter in $[\mathrm{mm}]$

$\mathrm{d}_{\mathrm{i}}=$ actual diameter in [mm] of shaft bore

$\mathrm{d}_{0}=$ outside diameter in [mm] of shaft. If the bore of the shaft is $\leq 0.40 \times \mathrm{xd}_{0}$, the expression

$1-\frac{d_{i}^{4}}{d_{0}^{4}}$ may be taken as 1.0

$\mathrm{F}=$ factor for type of propulsion installation

$F_{1}=95$ for intermediate shafts in turbine installation, diesel installations with hydraulic (slip type) couplings, electric propulsion installations

$F_{2}=100$ for all other diesel installations and all propeller shafts

$k=$ factor for the particular shaft design features, see M68.6

$\mathrm{n}_{0}=$ speed in revolutions per minute of shaft at rated power

$\mathrm{p}=$ rated power in $\mathrm{kW}$ transmitted through the shaft (losses in gearboxes and bearings are to be disregarded)

$\sigma_{B}=$ specified minimum tensile strength in $\mathrm{N} / \mathrm{mm}_{2}$ of the shaft material, see M68.3 
"Mircea cel Batran" Naval Academy Scientific Bulletin, Volume XIX - 2016 - Issue 2

The journal is indexed in: PROQUEST / DOAJ / Crossref / EBSCOhost / INDEX COPERNICUS / DRJI / OAJI I JOURNAL INDEX I I2OR / SCIENCE LIBRARY INDEX / Google Scholar / Academic Keys/ ROAD Open Access I Academic Resources / Scientific Indexing Services / SCIPIO / JIFACTOR

The diameter of the propeller shaft located forward of the inboard stern tube seal may be gradually reduced to the corresponding diameter required for the intermediate shaft using the minimum specified tensile strength of the propeller shaft in the formula and recognizing any limitations given in M68.3. [4]

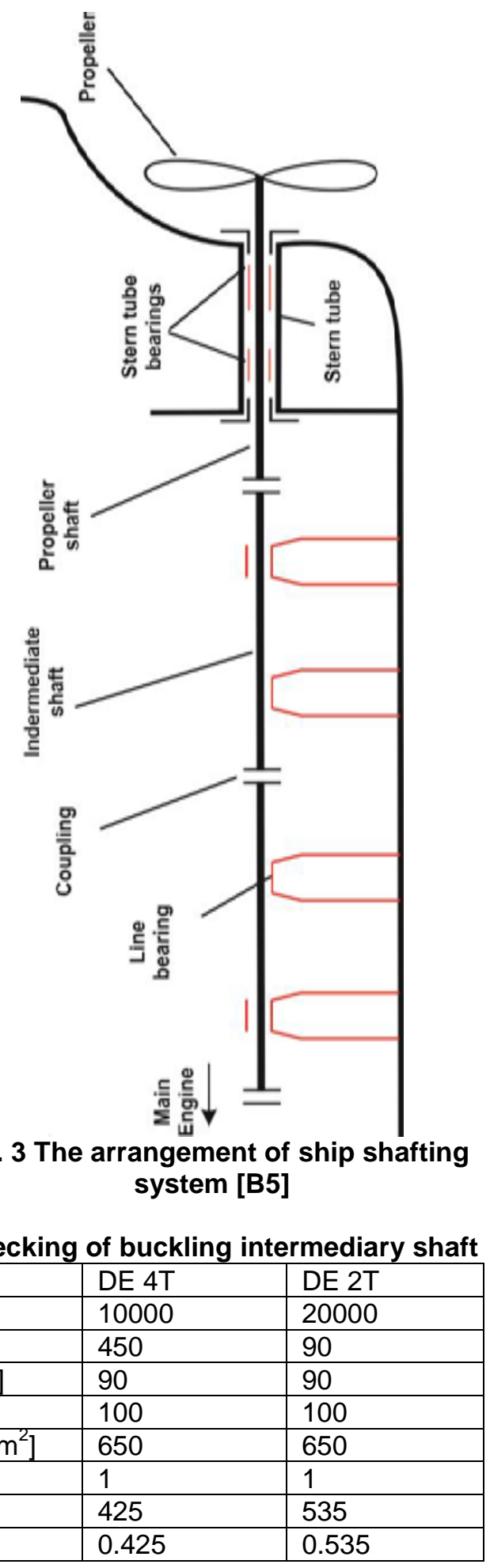

\begin{tabular}{|l|l|l|}
\hline $\mathrm{L}[\mathrm{m}]$ & 8.5 & 10.7 \\
\hline $\mathrm{M}_{\mathrm{m}}[\mathrm{kNm}]$ & 1061 & 2122 \\
\hline $\mathrm{W}_{\mathrm{n} 1}[\mathrm{Nd}]$ & 20 & 20 \\
\hline $\mathrm{W}_{\mathrm{n} 1}[\mathrm{~m} / \mathrm{s}]$ & 10.29 & 10.29 \\
\hline $\mathrm{F}_{\mathrm{A} 1}[\mathrm{kN}]$ & 972 & 1944 \\
\hline $\mathrm{F}_{\mathrm{cr}}[\mathrm{kN}]$ & $1.84 \times 10^{5}$ & $2.91 \times 10^{5}$ \\
\hline $\mathrm{C}_{\mathrm{f} 1}[-]$ & 189 & 150 \\
\hline $\mathrm{W}_{\mathrm{n} 2}[\mathrm{~m} / \mathrm{s}]$ & 2 & 2 \\
\hline $\mathrm{F}_{\mathrm{A} 2}[\mathrm{kN}]$ & 5000 & 9487 \\
\hline $\mathrm{C}_{\mathrm{f} 2}[-]$ & 37 & 31 \\
\hline $\mathrm{W}_{\mathrm{n} 3}[\mathrm{~m} / \mathrm{s}]$ & 0.5144 & 0.5144 \\
\hline $\mathrm{F}_{\mathrm{A} 3}[\mathrm{kN}]$ & $1.944 \times 10^{4}$ & $3.69 \times 10^{4}$ \\
\hline $\mathrm{C}_{\mathrm{f} 3}[-]$ & 9.46 & 7.88 \\
\hline
\end{tabular}

E.2. Checking of buckling intermediary shaft

\begin{tabular}{|c|c|c|}
\hline & GT & ST \\
\hline $\mathrm{Pe}_{\mathrm{e}}[\mathrm{kW}]$ & 30000 & 60000 \\
\hline $\mathrm{n}$ [rpm] & 5000 & 3000 \\
\hline $\mathrm{n}_{0}[\mathrm{rpm}]$ & 100 & 100 \\
\hline $\mathrm{F}$ & 95 & 95 \\
\hline$\sigma_{\mathrm{B}}\left[\mathrm{N} / \mathrm{mm}^{2}\right]$ & 650 & 650 \\
\hline $\mathrm{K}$ & 1 & 1 \\
\hline $\mathrm{d}[\mathrm{mm}]$ & 592 & 746 \\
\hline $\mathrm{d}[\mathrm{m}]$ & 0.592 & 0.746 \\
\hline $\mathrm{L}[\mathrm{m}]$ & 11.84 & 14.92 \\
\hline $\mathrm{M}_{\mathrm{m}}[\mathrm{kNm}]$ & 2865 & 5730 \\
\hline $\mathrm{W}_{\mathrm{n} 1}[\mathrm{Nd}]$ & 20 & 20 \\
\hline $\mathrm{W}_{\mathrm{n} 1}[\mathrm{~m} / \mathrm{s}]$ & 10.29 & 10.29 \\
\hline $\mathrm{F}_{\mathrm{A} 1}[\mathrm{kN}]$ & 2916 & 5831 \\
\hline$F_{\text {crf }}[\mathrm{kN}]$ & $3.56 \times 10^{5}$ & $5.68 \times 10^{5}$ \\
\hline $\mathrm{C}_{\mathrm{f1}}[-]$ & 122 & 96.55 \\
\hline $\mathrm{W}_{\mathrm{n} 2}[\mathrm{~m} / \mathrm{s}]$ & 2 & 2 \\
\hline $\mathrm{F}_{\mathrm{A} 2}[\mathrm{kN}]$ & 15002 & 30000 \\
\hline $\mathrm{C}_{\mathrm{f} 2}[-]$ & 23 & 19 \\
\hline $\mathrm{W}_{\mathrm{n} 3}[\mathrm{~m} / \mathrm{s}]$ & 0.5144 & 0.5144 \\
\hline $\mathrm{F}_{\mathrm{A} 3}[\mathrm{kN}]$ & $5.832 \times 10^{4}$ & $1.166 \times 10^{5}$ \\
\hline $\mathrm{C}_{\mathrm{f3}}[-]$ & 6.10 & 4.867 \\
\hline
\end{tabular}

$P_{e}[k W]$ - effective power; $n$ [rpm] -RPM of thermal machinery; $\mathrm{n}_{0}$ [rpm] - RPM of intermediary shaft; $F$ - factor for type of propulsion installation; $\sigma_{B}[\mathrm{~N} / \mathrm{mm} 2]$ - specified minimum tensile strength in $\left[\mathrm{N} / \mathrm{mm}^{2}\right]$ of the shaft material; $\mathrm{K}$ - factor for the particular shaft design features; $d$ [m] - designed diameter of intermediary shaft;

$d=F \cdot K \cdot\left[\frac{p_{g}}{n_{0}} \times \frac{560}{\sigma_{B}+160}\right]^{\frac{1}{3}} \cdot 10^{-a}[\mathrm{~m}] ;[20]$

$L[\mathrm{~m}]$ - length of intermediary shaft;

$\mathrm{Mm}[\mathrm{kNm}]$ - torque;

$P=M \cdot \frac{\pi m_{0}}{30}[k W] ;[21]$

$W_{n}[N d]$ - ship speed; $F_{A 1}[k N]$ - axial force in shaft;

$P=F_{A 1} \cdot W_{N 1}[k W] ;[22]$

$F_{\text {crf }}[\mathrm{kN}]$ - critical force buckling;

$F_{c r f}=\frac{\pi^{2} \cdot \mathbb{E} \cdot I_{\min }}{\left(\frac{L}{2}\right)^{2}}[\mathrm{kN}] ;[23]$ 
"Mircea cel Batran" Naval Academy Scientific Bulletin, Volume XIX - 2016 - Issue 2

The journal is indexed in: PROQUEST / DOAJ / Crossref / EBSCOhost / INDEX COPERNICUS / DRJI / OAJI I JOURNAL INDEX I I2OR / SCIENCE LIBRARY INDEX / Google Scholar / Academic Keys/ ROAD Open Access I Academic Resources / Scientific Indexing Services / SCIPIO / JIFACTOR

$I_{\min }=\frac{\pi \cdot d^{4}}{64}\left[m^{4}\right] ; \quad[24]$

$\mathrm{C}_{\mathrm{f} 1}[-]$ - buckling safety factor; $\mathrm{W}_{\mathrm{n} 2}[\mathrm{~m} / \mathrm{s}], \mathrm{W}_{\mathrm{n} 3}$

[m/s] - maneuvering speed vessel; $F_{\mathrm{A} 2}[\mathrm{kN}], \mathrm{F}_{\mathrm{A} 3}$

\section{CONCLUSIONS}

1. Rods pistons and connecting rods are cylindrical bar;

2. The diameter of the piston rod of $B$ and the diameter of connecting rod of $C$ may be increased by $15 \div 30 \%$;

3. In the calculation of buckling hasn't were taken into account channels for oil circulation of piston rod used for piston head cooling; respective circulation channels of connecting rod used for lubricating oil from the camps crosshead to camp crankpin.

4. To avoid increased pressure engine cylinder must be adjusted injection system in accordance with procedures.

5. Periodic the safety valve mounted on the cylinder head must be checking and regulation;

6. Engine start be made with open purges;

7. Intermediate shafts are supported by radial bearings - buckling calculation must consider the distance between bearings;

8. Shaft calculation must be completed with torsional and axial vibration testing for propulsion power plant.

\section{BIBLIOGRAFY}

[1] http://www.mec.tuiasi.ro/RM2/capitole/Cap1.html

[2]http://www.rmcet.com/lib/Resources/E-Books/Mech-auto/E-Books\%20Machine\%20Design-

Khurmi\%20R.s/CHP-16.pdf

[3] http://www.marinediesels.info/4_stroke_engine_parts/The_4_stroke_Con_Rod.htm

[4] UR_M68_Corr1_pdf1802.pdf IACS Req.2005/Corr.1 2012

[5] hull_deformation_korbetis.pdf Effects of Hull Deformation on the Static Shaft Alignment Characteristics of VLCCs: A Case Study

[6] Pounders Marine Diesel Engines and Gas TurbinesEighth edition 2004 